La gestion éco-touristique de la vallée de Whakarewarewa en Nouvelle-Zélande : promotion ou subversion de la culture māorie?

\title{
Stéphanie Bory
}

\section{(2) OpenEdition}

Journals

Édition électronique

URL : http://journals.openedition.org/elohi/489

DOI : $10.4000 /$ elohi.489

ISSN : 2268-5243

Éditeur

Presses universitaires de Bordeaux

\section{Édition imprimée}

Date de publication : 1 janvier 2015

Pagination : 101-120

ISBN : 979-10-300-0044-3

ISSN : 2431-8175

Référence électronique

Stéphanie Bory, « La gestion éco-touristique de la vallée de Whakarewarewa en Nouvelle-Zélande promotion ou subversion de la culture māorie ? », ELOHI [En ligne], 7 | 2015, mis en ligne le 01 janvier 2016, consulté le 20 avril 2019. URL : http://journals.openedition.org/elohi/489 ; DOI : 10.4000/ elohi.489 


\section{La gestion éco-touristique de la vallée de Whakarewarewa en Nouvelle-Zélande : promotion ou subversion de la culture māorie?}

\section{STÉPHANIE BORY}

Institut d'Études Transtextuelles et Transculturelles,

Université Jean Moulin-Lyon

Les îles néo-zélandaises furent découvertes en 1642 par Abel Tasman, mais cartographiées seulement en 1769 par le navigateur britannique James Cook. Les premiers colons britanniques s'installèrent dans ce nouveau territoire dans les années 1830, et en février 1840 fut signé le Traité de Waitangi entre William Hobson, alors Consul pour la Couronne en Nouvelle-Zélande et 40 chefs māoris, ce qui plaça les terres néo-zélandaises sous souveraineté britannique et en fit une colonie. La Nouvelle-Zélande conserva ce statut jusqu'en 1907, date à laquelle elle devint un dominion au sein de l'Empire, tout comme l'Australie et le Canada, obtenant ainsi une plus grande autonomie. Elle put siéger aux Nations-Unies à partir de 1945. Ce n'est qu'en 1947 que la Nouvelle-Zélande accepta de ratifier le Statut de Westminster, pourtant voté par le Parlement britannique seize ans plus tôt et établissant que toute loi adoptée à Londres ne concernerait dorénavant plus les dominions sans leur demande ou leur consentement ${ }^{1}$ (Torrent: 68). Le pays progressait ainsi sur la voie de l'indépendance, progressivement acquise, sans véritable proclamation officielle ${ }^{2}$.

1. Le Statut de Westminster, adopté le $1^{\text {er }}$ décembre 1931, stipule : « No Act of Parliament of the United Kingdom passed after the commencement of this Act shall extend or be deemed to extend, to a Dominion as part of the law of that Dominion, unless it is expressly declared in that Act that that Dominion has requested, and consented to, the enactment thereof ", in Mélanie Torrent, British Decolonisation (1919-1984). The politics of power, liberation and influence, Paris, PUF, 2012, p. 68.

2. Voir www.nzhistory.net.nz/politics/milestones, consulté en juillet 2015. 
La signature, en février 1840, du Traité de Waitangi, entre de nombreuses tribus māories et les Britanniques, communément appelés «Pakehas " (Stafford 1976 : 11), officialise la création de la Nouvelle-Zélande. Pour les Māoris, la protection de leurs ressources naturelles constitue, alors, un élément fondamental du traité, ce qui n'est pas forcément clair pour les Britanniques (Young: 63). Quand la Couronne garantit la "possession paisible » de leurs "propriétés " (Traité de Waitangi : article 2) $)^{3}$, la version māorie du traité, signée par les chefs, assure la pleine autorité sur les trésors des terres et des mers. De la même manière, quand les Māoris pensent accorder la gouvernance du pays aux Britanniques, ce qui leur aurait permis de gérer leurs propres affaires, ils donnent en fait la souveraineté pleine et entière (idem, article 1). En effet, le terme « souveraineté » n'existant pas dans la langue māorie, le traducteur (d'origine anglaise) choisit, lors des négociations, un mot équivalent à "gouvernance", terme beaucoup moins fort que " souveraineté » (Ibid.). Ces problèmes de compréhension et de traduction ont entraîné des conflits qui perdurent encore aujourd'hui ${ }^{4}$, notamment sur la possession des terres. Un accord majeur, communément appelé Treelords, a été signé le 25 juin 2008, au Parlement de Wellington, afin de rendre à sept tribus māories 176000 hectares de forêts dans le centre de l'île du Nord. Le montant de l'opération s'élève à environ 200 millions d'euros 5 .

Les deux peuples collaborèrent pour créer en 1887 le premier parc national néo-zélandais, Tongariro (Young : 80). Le parc est le résultat d'un don réalisé par une tribu māorie afin de protéger plusieurs lieux sacrés contre l'appropriation, et surtout l'exploitation, des terres par les colons britanniques (Easton : $5-6)^{6}$. Il s'agissait de préserver le site et d'obtenir la reconnaissance, par les

3. Voir www.nzhistory.net.nz/politics/treaty/read-the-treaty/english-text, consulté en juin 2015.

4. Le musée de Waitangi, au nord d'Auckland, illustre ces conflits en présentant des décisions de justice prises par le Tribunal de Waitangi, créé en 1975 et constitué à $50 \%$ de Pakehas et 50 \% de Māoris. Il recense, en mars 2015, plus de 2000 plaintes de familles et de tribus māories. Voir également Martin Doutré, The Littlewood Treaty. The True English Text of the Treaty of Waitangi, Auckland, De Dannan Publishers, 2005.

5. Voir «Des Maoris indemnisés par la Nouvelle-Zélande », Le Monde, 26-06-2008 (sans nom d'auteur) : «Lors de la signature de ce traité, Tumu Te Heuheu, chef principal des Maoris, a expliqué que son objectif était de fournir aux jeunes ainsi qu'aux générations futures «une forte et durable économie». «C'est l'héritage que nous leur laissons», a-t-il ajouté. ».

6. Brian Easton, dans son ouvrage Towards a Political Economy of New Zealand, décrit l'arrivée au $\mathrm{XIX}^{\mathrm{e}}$ siècle des immigrants européens ainsi: «The first European political economy was not a settlement one, but that of the quarry. Rather than what the French described as a "colony of permanence", initially we were a "colony of exploitation". Its early economic viability depended upon the depletion of natural resources: whales, seals, native 
colons, de son importance pour les communautés locales. Ce fut une initiative de la tribu Ngati Tuwharetoa et de son chef Horonuku Te Heuheu qui fit don des trois sommets volcaniques qu'il décrivait ainsi : " un lieu sacré pour la Couronne, un cadeau éternel de ma part et de celle de mon peuple» (Young : 102 ; ma traduction). Pourtant, le rapport que les deux communautés entretiennent avec la nature reste fort différent, comme le souligne Bill Pearson dans son essai "Fretful Sleepers : A Sketch of New Zealand Behaviour and Its Implications for the Artist ", publié en 1952 dans la revue littéraire trimestrielle Landfall: "We haven't made friends with the land. We use it as a convenience, an expedient : no farmer that I know draws breath with a change of light on the foothills, sieves the earth through friendly fingers. If he did he wouldn't let it run wild with gorse and blackberries, then cruelly put a match to them regardless of soil erosion. " (Pearson : 28) Selon Pearson, les Māoris ont établi une relation fusionnelle avec la nature, contrairement aux Européens (28). Ernst Dieffenbach, scientifique allemand qui est allé vivre et travailler en Nouvelle-Zélande, surnomme les Māoris «les enfants de la nature " (Dieffenbach : 391). Ils sont, donc, davantage attachés à la nature, associée à de nombreuses légendes.

Cette relation privilégiée entretenue par les communautés locales avec leur environnement naturel est particulièrement frappante dans la vallée géothermale de Whakarewarewa, dans la région volcanique de Taupo. Whakarewarewa abrite environ 500 sources, la plupart étant des sources chaudes alcalines, et au moins soixante-cinq geysers, dont sept actifs ${ }^{7}$. Les Māoris possèdent de nombreuses croyances liées à l'activité géothermique, ainsi cette légende associée au geyser Pohutu :

In our culture, geysers such as Pohutu are viewed as gifts from the gods. Local belief is that Te Whakarewarewa Thermal Valley in which Pohutu Geyser lives, was formed when two sisters, Te Pupu and Te Hoata (known as the Goddesses of Fire), travelled beneath the earth while searching for their brother. As they got close to him, the sisters would lift their heads above the surface - creating geysers and other geothermal hotspots along the way ${ }^{8}$.

timber, kauri gum and gold and other minerals. It is a world in which the trader comes, exploits, and goes, leaving behind debris and ruins», pp. 5-6.

7. Dont le nom complet est Te Whakarewarewatanga O Te Ope Taua A Wahiao, signifiant «le soulèvement des guerriers de Wahiao » (www.rotorua.nz.com/whakarewarewa. aspx, consulté en mai 2014).

8. Cette description est donnée sur le site Internet de Te Puia, lieu touristique situé à Rotorua, dans la vallée géothermique, étudié plus loin dans cet exposé. Voir www. tepuia.com, consulté en mai 2014. 
Le vocabulaire utilisé dans cette citation montre de quelle façon les Māoris personnifient le geyser qui "vit» dans la vallée thermale, l'activité géothermique à Whakarewarewa étant provoquée par les pérégrinations de deux déesses sœurs.

La principale ville de la vallée est Rotorua, située au cœur de l'île du Nord, à $230 \mathrm{~km}$ au sud d'Auckland et considérée comme le lieu de naissance du tourisme néo-zélandais (Young: 99). La population est composée à $34,3 \%$ de Māoris, contre 14,9 \% à l'échelle nationale, selon les chiffres du dernier recensement réalisé en 2013 (Statistics New Zealand: 5), et comme l'indique la municipalité de Rotorua : « Rotorua est un district biculturel avec une population de plus en plus multiculturelle. Rotorua est le cœur du peuple Te Arawa et de la culture māorie en Nouvelle-Zélande » (Rotorua District Council : 1 ; ma traduction). C'est, de ce fait, une région particulière dans le pays, ce que souligne Jan Corbett dans un article publié dans le New Zealand Herald le 20 janvier 2001 et intitulé "Rumblings in Geyserland»: "Aside from the city's pungent sulphuric aroma, which locals don't notice, Rotorua's most striking feature is the equally strong Maori presence. Not only has the tourist industry relied on Maori culture as an integral part of the tourist experience, but here Maori comprise about $30 \%$ of the population, compared with $14 \%$ nationally. "



Carte de Rotorua

Source : http://rotorua-education.co.nz/wp-content/uploads/ 2009/11/rotorua-location-map.jpg, consulté en juin 2015. 
Il convient, donc, de s'interroger sur l'exploitation touristique du site naturel par les Māoris et les colons européens, depuis l'arrivée de ces derniers : les Māoris ont-ils développé l'écotourisme avant l'heure? Les ressources engendrées par cette activité leur permettent-elles réellement d'être autonomes et seuls maitres de leurs décisions, ou doivent-ils toujours se plier aux demandes et exigences d'acteurs extérieurs? Il s'agira d'étudier, dans un premier temps, le passé touristique de la vallée, parcourue par de nombreux visiteurs dès le $\mathrm{XIX}^{\mathrm{e}}$ siècle, afin de voir comment Māoris et Pakehas ont exploité l'environnement naturel. Puis il sera intéressant de se pencher sur l'activité touristique actuelle pour se demander dans quelle mesure l'écotourisme peut être une manne pour les communautés indigènes locales ou, finalement, une nouvelle forme de colonialisme.

\section{Une longue tradition touristique : de l'écotourisme avant l'heure?}

Les premiers habitants de la région ont été les Māoris de la tribu («iwi») Ngati Whakaue, arrivée en Nouvelle-Zélande à bord de la pirogue Te Arawa, dont le nom est souvent utilisé de façon générique pour désigner les Māoris installés autour de Rotorua depuis près de 700 ans, descendants du chef Tamatekapua9. Whakarewarewa est le site de l'ancienne forteresse māorie ( $p a »)$ de Te Puia, datant de 1325. Les Māoris ont, donc, les premiers occupé ce territoire, tirant avantage de l'activité géothermique pour se chauffer et cuisiner ${ }^{10}$. Les premiers colons n'arrivèrent dans la région que dans les années 1830, et en petit nombre. Mais ils prirent très vite conscience de l'attrait de la vallée qui devint un site convoité et acquit une importance particulière pour les deux peuples ${ }^{11}$.

Whakarewarewa a un intérêt touristique et donc économique pour les Māoris et les Pakehas qui comprirent très vite les bénéfices potentiels à tirer de la région, ce que souligne Jenny Haworth dans Destination Rotorua, publié en 1995 : "Rotorua is intriguing - a lakeside city that rides on its own geothermal basin, a place where geysers and mud pools bubble close to the centre of the city. [...] Both Maori and Pakeha have long appreciated the warmth of Rotorua's thermal pools. » (Haworth, quatrième de couverture). Le site a été, de ce fait, très vite exploité : la ville, par les Européens, et les alentours, par les Māoris (Stafford 1988 : 9).

À l'époque victorienne, les cures thermales étaient à la mode et la région acquit rapidement une forte notoriété, après l'arrivée, en 1839, du premier

9. Voir www.tepuia.com/new-zealand/culture-architecture-and-pa, consulté en mai 2014.

10. Voir www.whakarewarewa.com/About+Us.html, consulté en mai 2014.

11. Ibid. 
«touriste", John Bidwill, décrit en ces termes par Don Stafford : «ce grand naturaliste qui, en plus d'avoir étudié les zones géothermiques de Rotorua, a acquis une certaine notoriété en devenant le premier homme (depuis Ngatoroi-rangi ?) à escalader Tongariro » (Stafford 1976: 9 ; ma traduction). John Bidwill publia, en 1841, un ouvrage, Rambles in New Zealand, qui ne manqua pas d'encourager de nombreux Européens à faire le voyage. Quatre guides furent édités entre 1872 et 1878 , le plus ancien encore conservé aujourd'hui s'intitulant Guide to the Hot Lakes, by H. G., publié comme une lettre adressée à l'éditeur du Tauranga Bay of Plenty Times, en août 1872 (Stafford 1976: 9). Il est agrémenté d'une publicité, en pleine page, pour un hôtel de Rotorua. La ville, ainsi que la région entière, devint, alors, un site thermal :

The potential value of Rotorua's thermal springs as a source of revenue has been noted as early as 1874. Concentrated not far from Lake Rotorua's edge is an area known as Te Kauanga, where a variety of thermal pools nestled amongst pumice, sulphur and manuka. When tourists arrived to see the Pink and White Terraces of Rotomahana they also wanted to bathe in this wild thermal area ${ }^{12}$.

Dès 1881, la ville est désignée tourist resort par le gouvernement britannique dans le cadre du Thermal Springs Districts Act, voté en septembre et le premier bâtiment thermal, appelé Pavilion Bath, ouvre l'année suivante ${ }^{13}$. Deux ans plus tard, elle obtint le statut de special town district pour promouvoir le potentiel de la ville en tant que destination thermale et les autorités décidèrent la construction d'un centre de cure, Rotorua's Bath House, qui fut inauguré le 13 août 1908, véritable symbole des richesses et revenus apportés par l'activité de la région. D'importants intérêts économiques étaient en jeu. La région se transforma en véritable centre touristique, comme l'envisageait, en 1908, T. E. Donne, alors Superintendant pour le Department of Tourist and Health Resorts: "The stream of travellers has certainly set towards NZ, and the establishment of up-to-date Government baths will no doubt make Rotorua world-famous and be the means of attracting many thousands more visitors yearly" ${ }^{\prime 1}$. De façon surprenante ce tout jeune ministère prend, en 1907, le contrôle total de la ville, alors petite communauté de 2000 personnes. La ville reste sous l'autorité du gouvernement britannique jusqu'en 1922. À la fin du xix ${ }^{e}$ siècle, les premiers touristes viennent

12. Les anciens bâtiments des thermes à Rotorua abritent aujourd'hui le musée de la ville. L'histoire de la municipalité est retracée sur son site Internet, notamment la tradition du thermalisme dans la région : www.rotoruamuseum.co.nz/visit-us/taking-thecure/the-spa, consulté en mai 2014.

13. Idem.

14. Voir http://www.rotoruamuseum.co.nz/visit-us/taking-the-cure/the-bath-house-story/, consulté en mai 2014. 
principalement admirer les Terrasses roses (Otukapuarangi, «fontaine du ciel nuageux ») et blanches (Te Tarata, "rocher tatoué »), considérées à l'époque comme la huitième merveille du monde, jusqu'à leur destruction en 1886 par l'éruption du volcan Mont Tarawera ${ }^{15}$. C'est, alors, l'attraction touristique la plus célèbre de Nouvelle-Zélande, attirant des Européens dès les années 1880, à une époque où le pays était peu accessible. Il fallait effectuer un très long voyage pour atteindre les Terrasses (bateau jusqu'à Tauranga, puis calèche, diligence, pirogue et marche). Comme cela a été dit, à l'époque, Pakehas et Māoris se partageaient l'exploitation des sites de la région.

Les Māoris, propriétaires du site et conscients des enjeux représentés par l'arrivée de touristes dans la vallée, se transformèrent très vite en magnats des affaires, ce que souligne Don Stafford, évoquant les années 1880 : «Regardless of the comments concerning social conditions at Te Wairoa village, the Maori people were well aware of the commercial advantage they held in being the owners of the Pink and White Terraces and they were determined to brook no rivals in their domain. » (Stafford 1976 : 26) Ils détenaient le monopole des visites, notamment des traversées en pirogue du lac Rotomahana pour atteindre les terrasses, faisaient payer les photographies et proposaient, contre quelques pièces, de faire la danse guerrière (le haka) : "Selon des études contemporaines, l'étalage d'indécence dans le haka augmentait en proportion directe avec la rémunération perçue » (Stafford 1976:26; ma traduction). Les femmes māories jouaient un rôle majeur dans cette activité touristique puisqu'elles faisaient office de guides, lançant ainsi une tradition qui perdure encore aujourd'hui (Ibid. : 28, Stafford 1988 : 52). Plusieurs sont restées très célèbres, à l'instar de Guide Sophia (Sophia Hinerangi, 1830/34 ?-1911), qui est considérée comme l'une des guides préférées des touristes à l'époque et décrite ainsi : « une femme des plus intelligente, agréable, qui parle remarquablement bien anglais. Elle est raffinée et possède des traits fins " (Ibid.; ma traduction). Après la destruction des terrasses en 1886, elle devint guide à Whakarewarewa puis, en 1896, gardienne de la réserve thermale qu'elle faisait visiter à des touristes prestigieux, voire royaux. Cette activité, très lucrative pour les femmes māories, se poursuivit au $\mathrm{xx}^{\mathrm{e}}$ siècle: "At Whakarewarewa, particularly during this century [20 $0^{\text {th }}$ century], the visitors' enjoyment has always been heightened by a group of remarkable women - the Maori guides - who have conducted millions of strangers through the thermal area. » (Stafford $1988: 52$ )

$\mathrm{Au} \mathrm{Xx}^{\mathrm{e}}$ siècle, deux femmes guides sont entrées dans l'histoire : Maggie Papakura et Rangi Dennan (Guide Rangi), cette dernière faisant visiter le site à Édouard, Prince de Galles, en avril 1920, au Roi Georges VI et son épouse

15. Voir www.rotoruamuseum.co.nz/visit-us/the-pink-white-terraces, consulté en mai 2014. 
Elizabeth, puis à la Reine Elizabeth II et son époux, le Duc d'Édimbourg, en janvier $1954^{16}$. Elle prit sa retraite en février 1966, après une carrière de plus de 40 ans.

Les Māoris semblent, par conséquent, avoir été des précurseurs de l'écotourisme, puisqu'en tant que premiers habitants de la vallée, ils ont su tirer profit de l'attrait représenté par les sites naturels, tout en promouvant leur propre culture. En effet, l'Assemblée Générale des Nations Unies, dans une résolution adoptée le 21 décembre 2012, définit l'écotourisme de la façon suivante : "L'écotourisme ouvre de vastes perspectives en termes de préservation, de protection et d'utilisation durable de la biodiversité et des aires naturelles en encourageant les populations locales et autochtones des pays d'accueil tout comme les touristes à préserver et respecter le patrimoine naturel et culturel. ${ }^{17}$

Même s'il n'y avait pas encore de véritable réflexion sur la biodiversité ou sur l'utilisation durable de la région, les Māoris tiraient parti des aires naturelles tout en faisant la promotion de leur culture, et les bénéfices récoltés permettaient aux tribus de subsister.

Les Européens ont eu, dès le xix ${ }^{\mathrm{e}}$ siècle, l'idée de préserver la région, comme à Tuhoe, lieu décrit en ces termes : « un lieu dans lequel la population autochtone pourrait se développer et où ses montagnes et forêts seraient préservées comme une future station touristique, les oiseaux endémiques des îles y seraient protégés vu qu'ils sont chassés d'autres régions par l'avancée de la civilisation » (Seddon, Carroll, in Young : 104 ; ma traduction). Selon David Young, dans Our Islands, Our Selves, une telle politique est une façon de préserver les tribus et l'environnement : « l'idée de préserver à la fois un peuple et son environnement naturel comme un musée vivant » (Young : 105 ; ma traduction). L'objectif était double: préserver les tribus māories mais aussi développer le tourisme. Les deux peuples réussirent à cohabiter dans la vallée, du fait de l'arrivée tardive et limitée des colons britanniques. Les terres n'étant guère fertiles ni propices à l'exploitation agricole, peu d'Européens se sont installés durablement. De plus, les chefs māoris ont su imposer leur autorité.

Il convient, à présent, d'étudier comment la vallée géothermale est exploitée aujourd'hui, afin de voir si le tourisme est réellement une manne pour les tribus māories ou une nouvelle forme de colonialisme.

Rotorua reçoit 1,2 million de touristes chaque année, dont les deux tiers sont néo-zélandais, alors que le pays compte moins de 4,5 millions d'habitants (Corbett). La vallée géo-thermale de la région de Rotorua est, à présent exploité, par

16. Une vidéo de l'événement subsiste aujourd'hui : http://www.nzhistory.net.nz/media/video/queens-visit-to-whakarewarewa, consulté en mai 2014.

17. Voir http://media.unwto.org/fr/press-release/2013-01-08/assemblee-generale-des-nations-unies-l-ecotourisme-joue-un-role-essentiel-d, consulté en juillet 2015. 
les Māoris sur deux sites : Te Puia et Te Whakarewarewa. Tous deux sont décrits comme des lieux culturels vivants : « une institution culturelle vivante ${ }^{18}$ pour le premier et, de même, un "village māori vivant $»^{19}$ pour le second. Ils ont, tous les deux, signé la charte du tourisme durable de Rotorua, initiative lancée en 2001 pour établir un groupe engagé dans le tourisme durable ${ }^{20}$. L'objectif est donc double : économique bien sûr, puisque les activités, sources de revenus, permettent à la tribu de subvenir à ses besoins, mais aussi culturel, par la transmission et la préservation de la culture māorie, notamment grâce au New Zealand Maori Arts and Crafts Institute, ouvert depuis $1963^{21}$. Il faut ajouter que l'activité touristique contribue à la préservation du site naturel : source de revenus, il n'a pas été livré au développement industriel, ce que confirme Michel Lextreyt «Thermalisme, manifestations volcaniques originales, contact avec la nature, découverte de la culture maorie : tous les ingrédients sont réunis pour faire de Rotorua une grande plate-forme touristique " (Lextreyt : 157).

Le premier site, Te Puia, situé à la sortie de la ville, fonctionne comme une véritable entreprise. Il emploie entre 85 et 120 personnes, selon la saison, dont 18 guides, tous māoris ${ }^{22}$. Au sein du comité de direction, cinq responsables, sur sept, sont également māoris. Le site accueille 400000 visiteurs par an, dont $90 \%$ sont étrangers ${ }^{23}$. Il est très complet puisque chaque visiteur peut non seulement admirer le geyser Pohutu, mais aussi voir le centre de formation de sculpture sur bois, le tressage des vêtements traditionnels, le Kiwi Centre ${ }^{24}$, avant de savourer un plat traditionnel (le hangi). De nombreux employés sont les descendants des premiers habitants établis dans la vallée $e^{25}$.

Te Whakarewarewa, à quelques kilomètres de Rotorua, est conçu de façon différente, puisqu'il s'agit d'un village dans lequel des Māoris vivent encore aujourd'hui, et qui ouvre ses portes aux touristes dans la journée. Dans un clip

18. «Te Puia is New Zealand's living Maori cultural centre», premiers mots de présentation sur le site www.tepuia.com/new-zealand/, consulté en mai 2014.

19. "The living Maori Village», comme indiqué sur la page d'accueil du site du village de Whakarewarewa, www.whakarewarewa.com/About+Us.html, consulté en mai 2014.

20. Voir www.sustainablenz.com, consulté en mai 2014.

21. www.tepuia.com/new-zealand/Overall-history/, consulté en mai 2014.

22. Ces chiffres m'ont été communiqués en juin 2014 par Denise Emery, responsable des Ressources Humaines à Te Puia, lors d'un entretien.

23. Ibid.

24. Il héberge un couple de kiwis, l'un des oiseaux endémiques de Nouvelle-Zélande, et contribue ainsi à la préservation de l'espèce en voie de disparition (Young : 229).

25. J'ai eu l'opportunité de visiter les lieux et de faire la rencontre de Kirimatao West, guide issue d'une longue lignée de guides dans la vallée, et soucieuse de faire partager ses traditions. Visite et entretiens informels ont eu lieu en mars 2014. 
promotionnel diffusé sur Youtube, Te Whakarewarewa insiste sur le fait que l'activité touristique permet de préserver le mode de vie traditionnel de la tribu locale : «Vous avez contribué à préserver un mode de vie unique ${ }^{26}$.

Ainsi, l'exploitation de la vallée de Whakarewarewa semble constituer un exemple réussi de tourisme durable, dans la mesure où l'équilibre entre protection de la nature et préservation de la culture est atteint dans une région dynamique, au demeurant. De nombreux visiteurs, notamment étrangers là encore, viennent admirer, en premier lieu, la beauté des sites naturels, ce qui leur permet également de découvrir la culture māorie et, ainsi, de contribuer à la préserver dans la mesure où elle est au cœur d'une activité commerciale créatrice d'emplois (entre 85 et 120 personnes seulement pour le site Te Puia, comme indiqué plus haut) et source de revenus. En effet, la population māorie est en progression dans la région : elle représente, aujourd'hui, $34,3 \%$ de la population de la ville de Rotorua, contre $30 \%$ lors du recensement de 2001. Cette progression devrait se poursuivre puisque 50,6\% des moins de 15 ans descendent de Māoris (Rotorua District Council : page iii). L'activité touristique permet à ces derniers de trouver du travail dans la région et, donc, de rester au sein de la tribu. Néanmoins, on peut se demander si les membres des communautés locales ne courent pas le risque de perdre leur identité, en se transformant en hommes d'affaires et en hommes de scène soucieux de plaire aux touristes.

\section{Les dangers de l'écotourisme}

Tout d'abord, peut-on concilier tourisme de masse et respect de la culture et de la nature ? Tourisme moderne et écotourisme ? En effet, compte tenu du nombre croissant de visiteurs dans la vallée de Whakarewarewa, il semble difficile de préserver les sites naturels et de conserver l'authenticité culturelle. Le tourisme de masse nécessite des infrastructures qui sont en contradiction avec le principe même de développement durable, ce que souligne Ngahuia Te Awekotuku, ellemême membre d'une tribu locale, dans son ouvrage The Sociocultural Impact of Tourism on the Te Arawa People of Rotorua, Ngahuia Te Awekotuku : « As Tupuni$u a$, and other writers previously mentioned, all warn, the avalanche of tourism that most Rotorua business folk are excitedly busying themselves for may well be that - an avalanche, ultimately damaging to the cultural and scenic uniqueness of the region. " (Te Awekotuku : 291) Elle encourage le développement d'un tourisme davantage personnalisé, destiné à une clientèle plus aisée, et non le tourisme de masse, caractérisé par des cars entiers de visiteurs, comme à Te Puia. Je prendrai

26. Voir www.youtube.com/watch ?v =O3ls0CRZvC8, consulté en mai 2014 ; ma traduction, pour le slogan. 
cet exemple, en guise d'illustration. Les visites sur le site se succèdent toutes les heures et sont, par conséquent, chronométrées, comme j'ai pu le constater par moi-même : 10 minutes devant les sculpteurs, 10 minutes devant les femmes qui tressent, etc. Il a même été suggéré d'utiliser un petit train pour transporter les touristes d'un geyser à l'autre! Certains sites, comme à Waitapu, déclenchent les geysers à heure fixe de façon artificielle, pour arranger les touristes et surtout les voyagistes qui peuvent, ainsi, planifier facilement les visites. Les guides euxmêmes ne s'en cachent pas, expliquant que, sans cela, les touristes se lasseraient d'attendre pendant des heures le déclenchement d'un geyser. Toute activité touristique est, certes, soumise aux lois de l'offre et de la demande, et il ne s'agit pas de considérer les touristes comme des agents d'un nouveau colonialisme, mais jusqu'à quel point les responsables des sites touristiques doivent-ils répondre à leurs demandes?

En outre, les spectacles proposés aux touristes, après la visite des geysers, s'apparentent davantage à une caricature folklorique, et sont fort éloignés de la réalité culturelle māorie, ainsi la cérémonie d'accueil, répétée chaque jour pour accueillir les touristes au sein de la tribu. Cette cérémonie, chez les Māoris, permet aux voyageurs arrivant dans une tribu de montrer que leurs intentions sont pacifiques en faisant une offrande au chef. C'est une façon de se présenter et de montrer que les nouveaux venus et les habitants appartiennent au même peuple. La gestuelle et les paroles sont, certes, celles utilisées depuis des décennies par les Māoris, mais cette cérémonie ne semble être qu'une représentation pour les besoins du spectacle et non une véritable reconnaissance entre membres d'une même communauté. Lorsque Michel Lextreyt évoque la pluri-ethnicité de la Nouvelle-Zélande, il souligne l'importance des racines māories, fort bien utilisées par l'industrie touristique : «Ces racines maories, on les retrouve dans quelques traditions plus ou moins bien sauvegardées qui nourrissent un folklore dont sait si bien se servir la promotion touristique " (Lextreyt : 173).

La polémique n'est pas récente puisque, depuis le début $\mathrm{du} \mathrm{xx}^{\mathrm{e}}$ siècle, trois projets controversés transformant le village en véritable site touristique furent proposés. Dès 1901 tout d'abord, afin d'exploiter au mieux le site géothermal et de préserver la culture autochtone, le Tourist Department recommandait de construire un village fortifié māori ( $p a h)$ modèle à Whakarewarewa, pour permettre aux visiteurs de mieux appréhender le mode de vie des Māoris avant l'arrivée des Européens. Des voix s'élevèrent contre un tel projet, jugé artificiel. Ainsi, cet extrait d'un article du Bay of Plenty Times, publié le 7 décembre 1904 : "The imitation Maori fighting pah now being erected by the Tourist Department near Whakarewarewa for the edification of globetrotters is pronounced by people who understand such things, to be a ludicrously feeble affair, no more like the real 
thing... than a green cheese is like the moon. » (BPT, 07-12-1904, cité par Stafford $1988: 51$ )

Un nouveau tournant se produisit dans les années 1960 : le site fut agrandi, puisqu'en 1963 fut lancé un projet de complexe touristique comprenant des bureaux, un grand atelier pour les apprentis sculpteurs sur bois, un lieu d'exposition pour leurs travaux et des infrastructures pour les guides. L'entrée devint payante. Le Maori Arts and Crafts Institute ouvrit ses portes, comme le rappelle Don Stafford :

One development was an innovative move because it not only introduced a new concept of tourism but acted as a spur to enthusiasm for Maori arts, crafts and culture. In June 1962 the Tourist and Publicity Department decided it was time for an upgrading of the whole area of Whakarewarewa under their control, which included the major thermal valley as well as the model pah at the western end. (Stafford 1988 : 329)

La polémique s'enflamma de nouveau dans les années 1980 lorsqu'un projet de reprise du site de Whakarewarewa par Maori International fut annoncé. Il s'agit d'une entreprise dont l'objectif est de regrouper de petites activités commerciales māories afin d'en favoriser le développement : « une entreprise visant à $[\ldots]$ agir comme un catalyseur pour le développement du peuple māori et de ses ressources " (Stafford 1988: 371 ; ma traduction). Les habitants ne furent pas consultés et, lors d'une réunion rassemblant, pour la première fois depuis 22 ans, l'ensemble des tribus de Te Arawa, ils décidèrent de rejeter le projet: "S'en suivit une plus grande consternation lorsqu'il fut révélé que leurs consultants hawaïens proposaient des attractions décrites dans la presse comme un style de développement à la Disneyland "(Stafford 1988:371; ma traduction).

Les communautés locales courent-elles, dans ces cas-là, le risque de perdre leur véritable identité ? La question mérite d'être posée, notamment en ce qui concerne les représentations culturelles proposées sur les deux sites. Ngahuia Te Awekotuku s'interroge sur les répercussions de l'activité touristique sur les habitants. Évoquant les spectacles proposés aux touristes, elle insiste sur la spécificité de telles performances, véritables représentations, différentes de la vraie culture : "Even the dancers themselves are aware of the difference - for the Maori, they present one thing, for the tourist, another, with no conflict, conscience or confusion. The tourist stage is work, the marae stage is tribal pride, and the latter is immeasurably more important. " (Te Awekotuku : 198) Elle établit donc une distinction très claire, ainsi qu'une hiérarchie, entre la culture elle-même et l'activité touristique. Elle insiste, néanmoins, sur le fait que les représentations ne vont pas à l'encontre des traditions māories, les deux aspects de la culture locale étant parfaitement conciliables. Son avis est partagé par Denise Emery, citée plus haut, qui indique dans un entretien accordé à l'auteur en juin 2014 : 
«Ils [les personnes se produisant] comprennent que c'est un spectacle pour les touristes. Mais les traditions et les chansons sont authentiques, nous n'inventons pas au nom du tourisme. Rien de ce que nous faisons dans la culture māorie n'est fait sans réfléchir et c'est en reconnaissance de nos ancêtres, nos traditions et notre histoire » (Emery; ma traduction).

Les spectacles culturels sont devenus de véritables shows, une culture spectacle, des reconstitutions éloignées de la réalité. Les Māoris qui participent à ces spectacles semblent se comporter comme des acteurs qui racontent une histoire dans le cadre d'une attraction, ce qu' indique le site internet même de Te Whakarewarewa: "Revivez l'histoire de notre village māori avec des histoires racontées par nos guides qui vivent ici depuis plus de cinq générations. Nous allons vous offrir une culture ne ressemblant à aucune autre et une attraction véritablement à ne pas rater ${ }^{27}$. L'utilisation du terme " attraction » montre que les participants font la différence entre la représentation artistique à l'attention des touristes et la culture, en tant qu'héritée des ancêtres et transmise de génération en génération.

Mais en va-t-il de même chez les spectateurs? Dans le clip vidéo présentant Te Whakarewarewa précédemment cité, un visiteur explique : «J'ai trouvé que c'était très authentique, c'était vraiment bien » (ma traduction), comme si les acteurs avaient bien joué... Les spectacles ne seraient alors qu'une mise en scène, destinée à amuser les touristes, et les habitants de simples statues d'un musée vivant. Les spectacles proposés par les Māoris n'offrent qu'une vision simplifiée, et forcément réductrice, de leur culture, riche et complexe. Ils ne proposent qu'un aperçu insistant sur les éléments devenus célèbres, à l'instar du haka qui, de nos jours, est associé à la culture māorie et en est devenu le symbole, voire le stéréotype... Les propos de Hywel Francis sur le développement excessif du tourisme au pays de Galles dans « A Nation of Museum Attendants " peuvent fort bien s'appliquer ici : «Le pays de Galles ne deviendrait-il rien de plus qu'une nation de gardiens de musée ? Mon cauchemar orwellien est un grand panneau noir faisant son apparition sur le Severn Bridge : «Vous pénétrez à présent dans des vestiges industriels protégés. Payez 5 livres sterling pour admirer la société en voie de disparition» » (Francis 1981; ma traduction). La région de Rotorua ne doit pas devenir un immense musée naturel où les habitants eux-mêmes feraient partie du paysage à admirer, et ne seraient qu'un élément du folklore. La description suivante conforte cette idée :

The central North Island with its spectacular thermal and volcanic features has long been a mecca for local and international tourists. They also visited Rotorua to see the

27. Voir www.whakarewarewa.com, consulté en mai 2014 ; ma traduction, pour la citation. 
'model Maori village' at Whakarewarewa. Ngati Whakaue and Tuhourangi people lived in the village, where some also worked as guides. [...] To create the impression of 'Maoriland' that some people expected, Maori dressed in traditional garments posed in 'daily activities' around the hot pools ${ }^{28}$.

De telles pratiques conduisent les nombreux visiteurs, notamment à Te Whakarewarewa, à oublier qu'ils ne se trouvent pas dans un simple musée ou dans un site entièrement sauvage, à tel point qu'ils ont parfois tendance à ne pas respecter la vie privée des habitants, n’hésitant pas à pénétrer dans des parties privées du site, ou à toucher ou ramasser des objets appartenant aux habitants ${ }^{29}$. Le Rotorua District Council a organisé, au début de l'année 1980, une enquête auprès des habitants de deux villages, Ohinemutu et Whakarewarewa, afin de connaître leurs attentes en termes de logement, d'infrastructures routières et récréatives, ainsi que d'aménagement. Quatre-vingt-deux questionnaires ont été distribués à Ohinemutu, sur lesquels soixante-neuf ont été complétés et retournés, soit un taux de retour de $88 \%$; et vingt-deux à Whakarewarewa, avec dix-sept réponses, soit un taux de retour de $77 \%$. Sur les 28 questions posées, trois concernaient le tourisme :

— «Question 20: Would you like to see more tourists visit your marae or village?»

— «Question 21: Do tourists create any problems in your village? If so, what would you like to see done to improve the situation? Keep tourists out? Restrict access? Provide walkway?"

— "Question 22: Are there any problems of privacy in your maraes?»

À Whakarewarewa, de nombreuses personnes interrogées se sont abstenues pour la question 20 , mais $63 \%$ de celles qui ont choisi de répondre ont accueilli favorablement l'idée de recevoir davantage de touristes. Le taux d'abstention montre que les habitants se sentent vraisemblablement gênés de devoir accepter de monnayer leurs traditions afin de maintenir leur mode de vie traditionnel. Les réponses à la question suivante sont plus nombreuses et $87 \%$ apportent une réponse affirmative. Enfin, $54 \%$ des répondants considèrent qu'il existe dans leur village des problèmes concernant la vie privée (Te Awekotuku : 145). Commentant cette enquête, Ngahuia Te Awekotuku montre l'ambivalence des Māoris qui souhaitent développer davantage le tourisme dans la région, tout en critiquant l'attitude de certains touristes : "The most common [reaction] insists

28. Voir www.nzhistory.net.nz/culture/living-in-the-20th-century/time-out, consulté en juin 2014.

29. Des habitants du village de Whakarewarewa m'ont fait part de leur sentiment, lors d'une visite en juin 2014, au cours de laquelle je les ai interrogés de façon informelle sur l'arrivée de nombreux visiteurs. 
that provided they are respectful and not a nuisance, tourists are all right. But too often they show little, or no, understanding of Maori values, and seem to assume that the village is here primarily for their own amusement. » (Te Awekotuku : 134) Une telle remarque indique que ceux-ci, aux yeux des Māoris, ne distinguent pas toujours représentation et réalité, ce qui n'est guère surprenant vu que les habitants se sont transformés eux-mêmes en acteurs. Le prix à payer pour préserver la culture locale serait alors la perte d'authenticité. Néanmoins, Te Puia et Te Whakarewarewa possèdent une histoire fort différente.

S'il existe, en effet, aujourd'hui deux sites à Whakarewarewa, ce n'était pas le cas jusqu'en 1997, date à laquelle une scission s'est produite à la suite d'un désaccord entre le gouvernement et la tribu Tuhourangi. Du jour au lendemain un portail fermé à clé est installé et coupe le village en deux sites : d'un côté Te Puia, qui correspond au complexe construit dans les années 1960 autour du village modèle, et de l'autre, le village de Whakarewarewa ${ }^{30}$. Finalement, dans la mesure où le village est le lieu où les membres de Te Arawa sont venus s'installer après l'éruption de Mount Tarawera à la fin du xix ${ }^{e}$ siècle, est-il resté plus authentique ? Propose-t-il aux visiteurs de se plonger dans l'atmosphère d'un véritable village māori, et non d'une reconstitution à but commercial ? C'est ce que considère Karen Shelford, qui possède et gère le site www.rotorua-travelsecrets :

Ever since the Whakarewarewa thermal valley was split down the middle in 1997, the foot traffic through Whaka, as we call it, slowed down. Possibly because of this, it has retained a sense of authenticity that can sometimes be taken as missing from Te Puia, next door. [...] What you get at Whaka today is still the village where the Tuhourangi/Ngati Wahiao people have lived for a hundred years now. They LIVE here. What you see is the real deal, not a reenactment of times gone by ${ }^{31}$.

Ce sentiment est partagé par Keith Weber, du Rotorua Group Events Pty MD, qui évoque ses souvenirs d'enfance dans un article publié par Dana Kinita dans le Rotorua Daily Post le 29 janvier 2014: "In 1940, as a 6-year-old Pakeha boy I would visit Rotorua and Whakarewarewa Village to cross the bridge and watch the Maori kids dive for pennies. In 1997 the gate between Te Puia and the village was locked separating the two attractions and preserving the village and the authentic lifestyle of Tuhourangi-Ngati Wahia. " Les habitants du village ont su préserver l'authenticité de leur culture en dépit de l'arrivée des touristes. Ainsi, le site de Te Whakarewarewa est plus authentique que celui de Te Puia, et relève

30. Voir www.rotorua-travel-secrets-com/rotorua-thermal-attractions, consulté en juin 2014.

31. Voir http://www.rotorua-travel-secrets.com/whakarewarewa-thermal-village.html, consulté en juin 2014. 
davantage de ce que devrait être l'écotourisme, selon la définition donnée par les Nations Unies.

L'activité touristique, dans la région de Rotorua, a, malgré tout, permis de préserver les traditions māories, ainsi transmises de génération en génération. Les danses et chants traditionnels sont appris par les jeunes générations, désireuses de bénéficier des ressources du tourisme. Ils auraient certainement en partie disparu sans cela. Pour conserver l'équilibre entre communautés locales et acteurs extérieurs non māoris, il est important que les Māoris puissent rester des acteurs de l'activité touristique et, surtout, des décisionnaires, non de simples danseurs ou chanteurs. C'est certainement le seul moyen de gérer efficacement l'impact environnemental, social et culturel du tourisme à Rotorua, en laissant les Mãoris prendre leurs propres décisions quant à l'utilisation de leur culture et de la nature qui les entoure. C'est aux communautés locales qu'il incombe de gérer l'activité touristique, en suivant les conseils de Herbert Hiller, spécialiste du tourisme, selon lequel : « For development, the guiding principle of tourism must not be 'you have to give them what they want', but instead, 'you have to give them what you have - and make what you have what they want'" (Hiller, cité par Te Awekotuku : 296).

Il est nécessaire que les Māoris de la vallée de Whakarewarewa ne deviennent pas les esclaves d'acteurs extérieurs. Nous ne pouvons que partager le sentiment de Ngahuia Te Awekotuku qui, après avoir participé, pendant deux ans, à l'activité touristique de sa propre tribu dans le cadre de ses recherches, a revu son point de vue sur l'impact du tourisme sur les communautés locales :

In the beginning, I set out to prove my personal belief that tourism had ruined Te Arawa, and blighted the Maori world generally. I was soon to discover that this was not so.

Instead of an exposure of negativity and defeat, this exercise became a celebration of resilience, and triumph, a record of how Te Arawa coped with colonization, then the neocolonization of tourism, and finally modern entrepreneurial commercialism, and coped well. (Te Awekotuku 283)

En différenciant clairement les représentations pour les touristes de la culture traditionnelle, en développant des pratiques touristiques plus intimistes, et en tenant les rênes de ce secteur économique, les Māoris pourront échapper aux dangers économiques, culturels et sociaux, que Te Awekotuku n'hésite pas à assimiler à du néo-colonialisme. Une étude menée en 2008 par le New Zealand Māori Tourism Council, association qui regroupe plusieurs centaines d'organisations touristiques régionales māories, révèle que $37 \%$ de ces organisations proposent des visites guidées, $15 \%$ un hébergement, $15 \%$ de l'artisanat, $12 \%$ des spectacles et $11 \%$ de la vente au détail. Les Māoris ont su diversifier leurs 
activités touristiques. Il s'agit aujourd'hui de les adapter à la culture locale, afin de ne pas proposer le même spectacle dans toute la Nouvelle-Zélande.

En conclusion, la vallée thermale de Whakarewarewa est exploitée depuis longtemps par les Māoris et les Pakehas qui ont compris les atouts de la région. Les visiteurs, attirés par la beauté du site et les bienfaits du thermalisme, représentent une source de revenus importante. Les communautés locales, dès le xix ${ }^{e}$ siècle, ont su tirer parti de cette manne, proposant leurs services comme guides et faisant découvrir leur culture. Elles ont ainsi pu mettre en valeur leur territoire et développer des revenus économiques. Néanmoins, l'essor de l'activité touristique dans la région de Rotorua menace d'être une nouvelle forme de colonialisme dans la mesure où les Māoris, confrontés à l'arrivée de visiteurs de plus en plus nombreux, se plient à leurs demandes et transforment leur culture en spectacle, susceptible de divertir le public. Tout en leur permettant de la préserver et de la promouvoir, l'éco-tourisme engendre aussi une perte d'authenticité.

Une vidéo promotionnelle de $2: 25 \mathrm{mn}$., réalisée à l'occasion de la sortie du film de Peter Jackson, The Hobbit et intitulée « $100 \%$ Middle Earth, $100 \%$ Pure New Zealand ${ }^{32}$, présente des sites touristiques majeurs du pays, dont Te Puia, et se termine par des images de Hobbiton, lieu de tournage du village des Hobbits à Matamata. Le titre même de cette vidéo est problématique car la Nouvelle-Zélande n'est pas la Terre du Milieu et ses habitants ne sont pas des Hobbits. Il est, d'ailleurs, possible d'acheter un billet combiné appelé «the Middle Earth Trilogy" pour visiter trois sites présentés dans cette vidéo : Te Puia, les grottes de Waitomo et Hobbiton. En quoi Te Puia fait-il partie de la Terre du Milieu ? Cette association purement commerciale a pour seul objectif de bénéficier du buzz autour de la nouvelle trilogie de Peter Jackson. Se priver de cette occasion de promotion serait déraisonnable pour le secteur touristique néozélandais, mais il serait dommageable de réduire la richesse du paysage à quelques images stéréotypées. Une telle vision réductrice du pays agace particulièrement les Néozélandais ${ }^{33}$. Il est indispensable de ne pas réduire les sites de Whakarewarewa au haka, aux danses et costumes, au hangi, et à Tolkien.

32. Voir http://www.youtube.com/watch?v=NhnSsM40loA, page consultée en mai 2014. 33. Trudy Agar, professeur à l'Université d'Auckland, m’a confié, lors d'un entretien en mai 2014, que la promotion excessive autour des deux trilogies réalisées par Peter Jackson, Le Seigneur des Anneaux et le Hobbit, permettait, certes, de promouvoir les paysages de son pays, mais tout en proposant une vision limitée, voire erronée. 
À l'heure actuelle, des discussions sont en cours pour réunifier les deux sites de Te Puia et Te Whakarewarewa ${ }^{34}$. En 2008, le gouvernement a rendu les terrains (70 hectares), sur lesquels Te Puia a été construit, au Whakarewarewa Joint Trust, composé de représentants des différentes tribus (Ngati Whakaue, Ngati Wahiao, Tuhourangi et Oruawhata). Depuis la constitution du Trust, le gouvernement les loue, pour exploiter le site dont il est toujours propriétaire. En juin 2014, les tribus locales ont décidé de négocier ensemble, avec le gouvernement, la restitution de Te Puia, même si l'attraction est gérée par des Mãoris. Elles souhaitaient obtenir un accord avant les élections législatives prévues en septembre 2014 mais, au moment de la rédaction de cet article, les discussions n'avaient pas encore abouti. La réunification des deux sites à Whakarewarewa, permettrat-elle un retour vers davantage d'authenticité, en couplant le village tel qu'il a toujours existé et les bâtiments ajoutés pour développer l'activité touristique?

\section{Bibliographie}

BIDWILL, John. Rambles in New Zealand. London, W. S. Orr \& Co., Paternoster Row, 1841, $34 \mathrm{p}$.

CORBETT, Jan. "Rumblings in Geyserland." New Zealand Herald. January $20^{\text {th }}, 2001$.

« Des Maoris indemnisés par la Nouvelle-Zélande », Le Monde, 26 juin 2008.

DIEFFENBACH, Ernst. Travels in New Zealand, vol. 1, London, J. Murray, $1843,452 \mathrm{p}$.

DOUTRE, Martin. The Littlewood Treaty. The True English Text of the Treaty of Waitangi. Auckland, De Dannan Publishers, 2005, 165 p.

EASTON, Brian. Towards a Political Economy of New Zealand. Dunedin, Hoken Library, 1994.

EMERY, Denise. Entretien accordé à l'auteure. Rotorua. Juin 2014.

FRANCIS, Hywel. "A Nation of Museum Attendants." Arcade. January 16th, 1981.

HAWORTH, Jenny. Destination Rotorua. Auckland, Penguin Books Ltd, $1995,33 \mathrm{p}$.

HILL, Richard S. Maori and the State: Crown-Maori Relations in New Zealand/Aotearoa, 1950-2000. Wellington,Victoria University Press, 2009, 364 p.

KINITA, Dana. "Rotorua Essential 50 : Day 48 - Whakarewarewa Living Village." Rotorua Daily Post. January 29 2014.

LEXTREYT, Michel. Nouvelle-Zélande, Aotearoa, le pays au long nuage blanc. Tahiti, Au Vent des îles, coll. «Terres d'Océanie », 2006, 222 p.

34. Voir http://www.rotorua-travel-secrets.com/whakarewarewa-thermal-village.html, consulté en juin 2014. 
PEARSON, Bill. Fretful Sleepers and Other Essays. London, Heinemann Educational Books, 1974, 168 p. Réédition de "Fretful Sleepers : A Sketch of New Zealand Behaviour and Its Implications for the Artist." Landfall, $\mathrm{n}^{\circ} 103$ (1952). Rotorua District Council. Changing Communities, Rotorua. Rotorua District Demographic Profile 2007. November 2007, 98 p.

Statistics New Zealand. 2013 Quick Stats about Māori. Wellington, New Zealand Government, 2013, $21 \mathrm{p}$.

STAFFORD, Don. The Romantic Past of Rotorua. Wellington, A. H. \& A. W. Reed, 1976, $72 \mathrm{p}$.

. The New Century in Rotorua: A History of Events from 1900. Rotorua, Rotorua District Council, 1988, 488 p.

TE AWEKOTUKU, Ngahuia. The Sociocultural Impact of Tourism on the Te Arawa People of Rotorua. Thèse de doctorat. Auckland, Université d'Auckland, 1981.

TORRENT, Mélanie. British Decolonisation (1919-1984). The Politics of power, liberation and influence. Paris, PUF, 2012, 304 p.

YOUNG, David. Our Islands, Our Selves. A History of Conservation in New Zealand. Dunedin, University of Otago Press, 2004, 298 p.

\section{Sites internet :}

- http://www.lemonde.fr/des-maoris-indemnisés-par-la-nouvelle-zelande, consulté en mai 2014.

- http://www.nzhistory.net.nz, consulté en juin 2014.

- www.nzhistory.net.nz/politics/treaty/read-the-treaty/english-text, consulté en juin 2015.

- http://www.rotoruamuseum.co.nz, consulté en mai 2014.

- http://www.sustainablenz.com, consulté en mai 2014.

- http://www.tepuia.com, consulté en mai 2014.

- http://www.whakarewarewa.com, consulté en juin 2014.

Résumé : Après avoir été, pendant plus d'un siècle, une colonie puis un dominion, au sein de l'Empire britannique, la Nouvelle-Zélande a progressivement acquis son indépendance, au cours du xxe siècle. Les Māoris, très attachés à la nature, ont tenté, depuis, de préserver leur culture et leur mode de vie traditionnel, notamment à Rotorua, dans la vallée thermale de Whakarewarewa, site exploité dès le XIXe siècle par les tribus māories locales et les colons britanniques. L'objet de cet article est d'étudier la longue tradition touristique dans la région, forme d'écotourisme avant l'heure, avant de s'interroger sur les dangers actuels de cet écotourisme qui permet, certes, de promouvoir la culture māorie, mais se révèle être, également, à l'origine de la subversion de cette même culture.

Mots-clefs : Nouvelle-Zélande, Māoris, Rotorua, écotourisme, Whakarewarewa, thermalisme.

Abstract: New Zealand, first conquered by Britain in the $18^{\text {th }}$ century, was a colony, then a dominion, in the British Empire for more than a century before it gradually obtained its independence in the $20^{\text {th }}$ century. The Māori community, deeply attached to nature, has since attempted to preserve its culture and traditional way of life, especially in Rotorua, located in Whakarewarewa Thermal Valley, a source 
of income ever since the $19^{\text {th }}$ century both for the local Mãori tribes and British settlers. This article aims to study how the area has long been a favoured tourist destination and resort, ran in a way announcing modern day ecotourism. It will then be considered whether ecotourism, contributing today to preserving the Māori culture, is not also a source of subversion of this very culture.

Keywords : New Zealand, Māoris, Rotorua, ecotourism, Whakarewarewa, thermalism.

Biographie : Stéphanie Bory est Agrégée de l'Université et Maître de Conférences en civilisation britannique, à I'Université Jean Moulin-Lyon 3. Elle est membre de I'Institut d'Etudes Transtextuelles et Transculturelles (IETT) et travaille sur l'écologisme et le nationalisme, notamment au pays de Galles. Après avoir soutenu une thèse sur la politique environnementale de l'Assemblée galloise, elle a publié différents articles sur ces sujets et participé à de nombreux colloques. Elle a organisé, en 2011, un colloque international sur la croissance verte dont elle prépare le second volet, prévu en 2016. Elle a co-organisé, avec Paloma Otaola, un colloque international sur le nationalisme au xxi ${ }^{e}$ siècle, en juin 2015, à Lyon.

Biography : Stéphanie Bory is a senior lecturer in British Civilisation in Jean Moulin-Lyon 3 University where she has been teaching since 2001, first to business students, then in the Law Faculty. She has been part of the Language Faculty since 2009. She is also a member of the Institute for Transtextual and Transcultural Studies and works on environmentalism as well as nationalism, especially in Wales. She obtained in 2008 a PhD on the environmental policy of the National Assembly for Wales. She has published articles, including one in Environmental Issues in Political Discourse in Britain and Ireland, recently published by CSP, and participated to conferences on these topics. She organised jointly with Paloma Otaola a conference on nationalism in the $21^{\text {st }}$ century in Lyon in June 2015. 